\title{
DSM-5 - Pros and Cons
}

The launch of DSM-III in 1980 triggered revolutionary changes in the field of psychiatry and associated sciences. The classification of mental disorders moved from partially arbitrary decisions to a reliable system. Before DSM-III, it was more likely that 2 diagnosticians resulted in different diagnoses for the very same patient than that they came to the same conclusion about the diagnostical label for the clinical problem. The introduction of a reliable classification system for mental disorders moved psychiatry, clinical psychology, and several other fields from low scientific recognition to one of the top positions of healthcare research. This also resulted in tremendous improvements in our understanding and treatment options for patients with mental disorders.

However, is DSM-5 still on this track of fostering research and treatment for mental disorders? Is science still the major purpose of DSM-5, or did it become the victim of economic interests and power of specific subgroups? The societal impact, but also the money that is made by DSM became tremendous, and this can threaten the scientific purpose. Just a small example: Authors are not allowed to cite DSM- 5 criteria for a single disorder without paying fees to the American Psychological Association (APA) press. This means that text books and other publications are not allowed to inform their audience about any DSM-5 diagnosis without paying for it. Is this the way how we want to disseminate scientific approaches? And the content of DSM-5, is it really based on the best of our knowledge? Critique has been expressed that some innovations are arbitrary and misleading [Rief and Martin, 2014].

As editor of the German journal VerHALTENSTHERAPIE (Behavior Therapy), I am proud that we were able to get 2 extremely distinguished experts of the field to discuss the pros and cons of DSM-5. Prof. Dr. Ulrich Wittchen (Technische Universitaet Dresden) was member of different DSM groups during the last 20 years, and no German scientist was more involved in this process. Prof. Dr. Allen Frances (Duke University School of Medicine, Durham, NC, USA) can be considered the main person who started the 'counterrevolution' against DSM: As chair of DSM-IV, he had the best insight into these processes, and he considers DSM- 5 as a misdevelopment that must be revised. $\mathrm{He}$ is our special guest author of this Pro-Con section, and we are extremely delighted to publish this discussion.

Winfried Rief, Marburg

\section{Reference}

Rief W, Martin A: How to use the new DSM-5 somatic symptom disorder diagnosis in research and practice: a critical evaluation and a proposal for modifications. Ann Rew Clin Psychol 2014; DOI: 10.1146/annurevclinpsy-032813-153745. 


\section{Contra}

\section{The Inclusion of Unsafe and Scientifically Unsound New Diagnoses Will Be Harmful and Could Entail Diagnostic Hyperinflation}

The international controversy surrounding DSM-5 has delivered a severe blow to its credibility and has also reduced the public's faith in the reliability and effectiveness of psychiatry. The inclusion within DSM-5 of unsafe and scientifically unsound new diagnoses will have harmful unintended consequences and threatens to turn our current diagnostic inflation into diagnostic hyperinflation. I will explain how DSM-5 went so far wrong, point out its worst dangers, and offer recommendations for minimizing them and for preventing similar disasters in the future.

\section{Excessive Ambition}

DSM-5 shot unrealistically high - early on it announced the goal of effecting a paradigm shift in psychiatric diagnosis. Two of its premature and failed ambitions - to include biological markers and dimensional ratings - were temporary distractions that looked silly but caused no permanent harm. The third - an attempt to promote preventive psychiatry by introducing new diagnoses and reducing thresholds for existing ones - will have potentially disastrous and long-lasting unintended consequences. An effective preventive psychiatry would have to meet 3 prerequisites: accurate diagnosis, effective treatment, and safety. None of the DSM-5 changes meets these standards. All will misidentify patients and result in excessive, often harmful treatments. Other medical specialities have experienced the dangers of premature preventive diagnosis and are tightening guidelines for screening and diagnosis - just as DSM-5 made the mistake of loosening them!

\section{Disorganized Methods}

There was little central direction of the DSM-5 work groups and insufficient quality control. As a result, the experts were given free rein to expand their pet diagnoses. Their proceedings were secretive, inflexible, and not open to outside influence and correction. Literature reviews were inconsistent in their quality and used very varying standards for making changes. Deadlines were always missed, often by more than a year.

\section{Sloppy Writing}

DSM-5 had no one experienced in writing diagnostic criteria and there was little text editing to ensure accuracy and consistency. As a result, DSM-5 is filled with egregious writing mistakes and ambiguous wordings that will make many of its diagnoses inherently unreliable and inaccurate [Frances, 2013].

\section{Publishing Profits Trump Public Trust}

The DSM-5 franchise has become a lucrative profit maker for the APA - vital to meet what would otherwise be a big budget deficit. From the start the workers on DSM-5 were forced to sign confidentiality agreements to protect the DSM-5 intellectual property - a decision that badly limited their interchange with the field. At the end, because of missed deadlines, DSM-5 was prematurely rushed to press in a raw and poorly edited state.

\section{Dangers}

In the USA, $25 \%$ of the population already qualify for a psychiatric diagnosis in any given year; $50 \%$ will face a psychiatric diagnosis in their lifetime. $20 \%$ of the population take psychotropic medication and $80 \%$ of the prescriptions are written by nonpsychiatrists with little training and an average of only 7 minutes to spend per patient. Drug company marketing has driven excessive medication use and there are now more deaths from overdose with prescription drugs than with street drugs. There have been false epidemics of Attention Deficit Disorder, Autism, and Childhood Bipolar Disorder. DSM-5 should have tightened the definitions of mental disorders and should have included cautions against the risks of careless diagnosis. Instead DSM-5 has introduced new disorders with a high prevalence (Minor Neurocognitive, Disruptive Mood Dysregulation, Binge Eating), has reduced the threshold of Attention Deficit Disorder, and has turned normal grief into Major Depressive Disorder. In aggregate, these changes can add tens of millions of new patients who will be misidentified at the fuzzy boundary with normality. None of the DSM-5 suggestions is supported by solid scientific data; none received a careful risk/benefit analysis; all represent the experts' enthusiasm to expand their pet areas of interest; and all are likely to result in much more harm than good. The furore over DSM-5 expansion of diagnostic boundaries by defining milder conditions has also distracted attention from a much greater problem facing psychiatry: the inadequate treatment of the severely mentally ill.

\section{What to Do}

My advice about DSM-5 is: don't buy it, don't use it, and don't teach it. It is an unfortunate aberration that needs correction. Hopefully, the International Classification of Diseases (ICD)-11 will learn the obvious painful lessons and will not fall into the same trap of expert arbitrariness and diagnostic exuberance.

\section{Future Revisions}

New diagnoses can do more harm than new drugs. The method of changing the diagnostic system needs to be changed. Diagnoses should be revised only when there is compelling evidence that any change is safe and scientifically sound. I have no confidence in the APA as the future steward of the diagnostic system. Its financial conflict of interest and 
proven incompetence require that it loses the DSM franchise. Unfortunately, the World Health Organization (WHO) is terribly under-resourced and the ICD-11 process also does not inspire confidence. Possible preferable alternatives might be something comparable to the vetting required before marketing new drugs or something resembling the Cochrane reviews. Allowing the experts in any given area to be ultimate arbiters is like letting the fox guard the henhouse. Decisions should also require input from experts in health economics, public health, primary care, and the public.

\section{Summary}

DSM-5 has created the worst crisis in confidence in psychiatry in 40 years. This should serve as a wake-up call. We need to provide much less care to those who do not really need it and should care much more for those with severe problems. If primary care doctors will be prescribing most of the psychotropic drugs, they need more training and need to spend more time doing it. It is very easy to give an inaccurate diagnosis, very hard to undo the harms caused. An accurate diagnosis can dramatically improve or even save a life. A misdiagnosis can haunt a person for a lifetime.

Allen Frances, Durham (NC)

\section{Reference}

Frances A: DSM-5 writing mistakes will cause great confusion. Huffpost Science 2013. http://m.huffpost.com/us/entry/3419747 (accessed 24.10.2013).

\section{+ Pro}

\section{DSM-5 Did Tighten Up the DSM-IV Criteria - Sensibly and in the Interest of Patients, Not of Politicians!}

Undoubtedly, the third revision of the Diagnostic and Statistical Manual of Mental Disorders (DSM-III) in 1980, with its core principle of explicit descriptive diagnostic criteria and diagnostic algorithms for mental disorders has significantly changed our field. What has been described in the 1970s as a largely idiosyncratic work of psychiatrists for psychiatrists with no reliability and cross-national consistency became a well-established and accepted base for all disciplines involved in the diagnosis and care for people with mental disorders [Phillips et al., 2003]. Subsequently, updated in a series of revisions (DSM-III-R, DSM-IV, and DSM-IV-TR), the DSM has also allowed the stringent derivation of improved methods (e.g., diagnostic instruments) and can be seen as a major promoter of impressive progress in research in psychology, psychiatry, and the neurosciences. To a certain degree it also unified research and demystified many potentially stigmatiz-
Table 1. Partly competing goals and functions of diagnostic classification systems (examples)

Ensuring communication amongst all groups (national and international) Relevance for all types of systems worldwide

Relevance for different orientations

Documentation

Reimbursement issues, legal issues

Utility for social, psychological, and neurobiological research

Clinical utility for diagnosis, prevention, treatment choice, and prognosis Acceptability for stakeholder groups

ing concepts of mental disorders. Thus DSM has helped patients and the public to develop a more appropriate understanding of mental disorders (e.g., 'you are not crazy - but you have a mental disorder for which effective treatments exist; you are not alone, up to one third of all people suffer from mental disorders at some point in their lives'). It is hard to imagine how all the progress regarding the causes, treatments, and consequences of mental disorders could have been made without the DSM approach. In fact, DSM-III and its revisions have significantly changed the way we deal with mental disorders and were also instrumental in making the European Union and governments around the world realize that mental disorders are the core healthcare challenge of the 21st century (table 1) [Wittchen et al., 2011].

A few months ago the fifth revision was published (DSM-5) associated with an unprecedented and unexpected broad media campaign criticizing the DSM-5 from various perspectives.

\section{Why Is DSM-5 Criticized?}

Before this question can be answered one has to remember that all revisions of diagnostic classification systems are inevitably and intimately tied to very different - and in fact competing - needs and interests of individuals and groups [Saxena et al., 2012]. We should also be aware that some critical voices might be motivated by personal reasons, such as researchers being frustrated by the fact of not having been asked to be actively involved in the revision process or stakeholders that have had bad experiences with mental healthcare. Thus, there is naturally a lot of room for dissatisfaction and criticism, with and without having actually examined the DSM-5. Beyond the general consensus that such systems should improve and ensure communication and a common language, the core principles of a good diagnostic classification system might be contradictory on many levels. This is especially true for research and treatment in the field of mental health, to which many disciplines with different traditions, methods, and approaches contribute by addressing partly very different patient populations. For example, physicians focusing on pharmacological treatments have diagnostic needs that differ from 
those of behavior therapists or psychoanalysts. Healthcare professionals dealing with substance abuse markedly differ in their concepts and the way they intervene from those taking care of patients with personality disorders. Neurobiologically oriented psychiatrists favor different models than behavioral psychologists. Primary care doctors might prefer simple diagnostic algorithms while various mental health specialist groups strive for higher degrees of differentiation emphasizing seemingly subtle, but conceptually and/or clinically highly meaningful distinctions. In the following, it is argued that DSM-5 is a necessary, timely, and important step forward. The evidence for this position is reviewed while addressing some of the key criticisms and taking into consideration the previous observations.

\section{The Revision Was Needed and in Fact Overdue}

A new revision of DSM, the standard reference in science and research of mental disorders accepted worldwide, was undoubtedly urgently needed and timely. Similar to other areas of medicine with high levels of scientific output and a rapidly increasing evidence base, the need for revisions is mounting whenever major advances in basic, preclinical, and clinical research necessitate adaptations or even major shifts in paradigms. From this perspective it is essential to stress that diagnostic systems by their very nature are always time-limited constructs - they are never final and perfect. They simply reflect the best possible international expert consensus regarding science and research evidence and issues concerning the provision of interventions in healthcare systems at a given point in time.

In the last decade, the progress achieved in research, particularly in psychology and the neurosciences, has started to exert considerable and still increasing pressure on the ICD and DSM to make significant and even 'revolutionary' changes in order to better reflect particular scientific needs. One example are the calls from many psychologists who strongly believe in dimensional and factor-analytic approaches for a dramatically simplified metastructure of 3-5 core dimensions of psychopathology [Wittchen et al., 2009]. Another example is as a recent neuroscience initiative of the National Institutes of Health (NIH) to implement Research Domain Criteria (RDoC) as a replacement of traditional diagnostic systems [Schumann et al., 2013]. Thus, several groups see sufficient evidence from their work for making major and even radical changes in the way we diagnose mental disorders, sometimes even promoting the development of alternative approaches to ICD and DSM.

Beyond all other potential motivations that might have played a role (including generating further income for the APA from distribution of the DSM-5), it is evident that the field had to react and to ensure that a common international language of psychopathology is retained and diagnosis of mental disorders in research and practice is consistent around the world. Ensuring common procedures and a common lan- guage regarding diagnostic labels, definitions, and rules allows improved communication and is regarded as the essential basis for and the link between research, education, everyday practice, and the healthcare system. But why DSM-5 and the APA? Why not leave this job to the WHO with its ICD? Well, the answer is simple: The WHO, by definition of its main objectives, is neither in the position nor able to fulfil this daunting task appropriately, especially for our field. Being the smallest common international political denominator of diagnoses used in - developed and less developed - healthcare systems across the globe the ICD has to be general rather than detailed, focusing on consensus of nomenclature and the crude scope as well as on structure of diagnoses. For historical reasons, the essential groundwork for this ongoing endeavour, such as the provision of specific criteria and rules, remains in the hands of the APA and DSM [Saxena et al., 2012]. They inform in a complementary way the forthcoming ICD-11 chapter for mental disorders, but not the other way around.

\section{DSM-5 Is a Significant Improvement over and beyond}

\section{$D S M-I V-T R$}

Does DSM-5 resolve any of the pressing core problems we still face in our field? Does DSM-5 resolve the problems of imperfect reliability and validity? Does DSM-5 come up with an improved and more systematic or even logical organization and classificatory principles? Does DSM-5 improve practice by providing better guidance for treatment or reducing the risks of potential harms? Does DSM-5 provide better guidance and promising methods for improved basic psychological and neurobiological research? Or does it improve significantly the utility for practitioners - whether primary care doctors or the various groups of mental health specialists? Disregarding the fact that in light of contradictory tasks and functions of diagnostic classification systems it would be inappropriate to evaluate the quality of the whole product by any single one of the aforementioned issues, we have to admit that we do not know the answers yet - only time will tell. Undoubtedly, even the most committed believers in DSM-5 will be unhappy with at least some features. Further, it is fair to state that many, particularly those neurobiological as well as psychological researchers hoping for a major and comprehensive paradigm shift in DSM-5, are disappointed - or should I say frustrated - for at least 2 main reasons:

First, rigorous examination of the diagnostic value of dozens of neurobiological paradigms and models in the process of the DSM-5 revision work clearly demonstrated that it is still unjustifiable and premature to use genetics or neuroimaging as diagnostic tools. Even the idea of unifying anxiety and stress-related disorders under a new umbrella of 'Stress- and Fear Circuitry Disorders' missed by far the bar for acceptance.

Second, despite all efforts, the stringent implementation of dimensional aspects and core overarching psychopathology dimensions as well as the introduction of an empirically based 
logical meta-structure was at best partially successful [Wittchen et al., 2010].

This 'failure', however, was not due to poorly organized or incompetent work groups and task forces of the DSM-5, but the documented outcome of a rigorous examination of research and clinical evidence by all involved in the development of DSM-5. The fact that DSM-5 contains fewer radical changes than expected and that the overwhelming majority of changes is minor, was for many sobering and frustrating. Nevertheless the sum of changes, and particularly the improved organization and consistency of the manual associated with a substantial reduction of pages and diagnoses will hopefully improve the utility and the frequency of its appropriate application in practice and research.

\section{The Revision Process Was of Unprecedented Quality and \\ Transparency}

The 5-year revision process was based on internationally balanced experts grouped by diagnostic domains. They identified critical issues and domains potentially in need for revision. In dozens of work groups and overarching task forces, they provided hundreds of systematic reviews as well as original and position papers regarding the state of the art. This process involved probably thousands of experts (e.g. nearly 200 in anxiety disorders alone), consultants, and advisors before suggestions for possible changes were made based on a formal process to examine scientific evidence and implications. In various rounds this evidence was discussed and evaluated before making the final proposals for a revision and eventually adopting them. Even more important was the much broader and systematic involvement of all stakeholder groups including patients, advocacy groups, healthcare providers, interest groups (insurances, forensic institutions, industry), and the public [Saxena et al., 2012]. All of them had almost unrestricted access to the carefully documented work group products and processes via the DSM-5 webpage. In fact, the DSM- 5 process could be described as an open access activity, because never before such a revision process capitalized that extensively on the efficient use of electronic media with all advantages and disadvantages. Finally, a series of general principles were agreed upon by consensus to ensure a rational decision regarding all potential revision points. In the initial stages, this process included propositions that were evidently obscure. Nevertheless, all propositions received serious and systematic scrutiny to ensure that changes not sufficiently supported by research evidence do not find their way into the DSM-5.

\section{DSM-5 - Driven by Interests of 'Big Pharma'?}

This complaint always seems to be a good sellout for the press - but is in fact of no substance. There was continuous, unprecedented scrutiny regarding the involvement of experts that might have a conflict of interests, concerning for example (but not limited to) industry. All advisors with significant potential conflicts of interests were excluded and all experts involved in the revision had to refrain from such activities during the revision process. More importantly: Is there currently really any such pressure? We all are aware, that most drug companies have greatly lost their interest in central nervous system (CNS) and mental disorder drug development and promotion over the last 10 years. The joint effects of loss of patent protection, high failure rates in comparison to other disease groups, and exceedingly high regulatory standards and costs have prompted big pharmaceutical companies to largely withdraw from CNS research. Some might say to a degree that one might be concerned, given the need for development of better pharmacological treatments.

It is certainly true that many of us are concerned about the degree of inappropriate treatment documented for several disorders, particularly regarding the inflationary drug treatment of so-called bipolar spectrum disorder and attention deficit / hyperactivity disorder (ADHD). However, is the DSM to be blamed for the potential misuse in cases where some clinicians seem to discard the existing diagnostic criteria and equate subthreshold symptoms as being an indication for drug treatment? Can a diagnostic system be held responsible for the misuse by certain interest groups?

\section{DSM-5 Does Not 'Invent' or 'Inflate' Diagnoses}

Some critics blame DSM-5 for applying psychopathological labels to normal everyday problems, 'inventing' new diagnoses, more generally promoting their inflationary use, and thus creating 'false epidemics'. This is simply wrong and unfounded for at least 3 evident reasons: (1) A count of diagnoses in DSM-5 reveals a lower number of diagnoses compared to DSM-IV and ICD-10. (2) Generally, stricter criteria are implemented, even for grief reactions, by adding a cautionary note, and (3) epidemiological modelling does not provide any evidence that DSM-5 may result in higher prevalence estimates. It is also important to note that not a single diagnosis was 'invented' or promoted by the DSM-5 work groups. All proposals came from the field, namely the users in practice and research settings, substantiated and weighted by research evidence, proof of utility, and appraisals of potential benefits and harms. So, the patients with their specific complaints are obviously real, together with research evidence for their condition as well as helpful treatment. However, as providers in our particular field of interest might not see them every day, they might not see the need for having them diagnosed. But does that justify not paying attention?

So why do such speculations flourish? I see this primarily as an 'unintended serious side effect' of the open and transparent DSM-5 revision process. In this process, particularly in the beginning, all formal proposals were registered before they were subjected to rigorous tests. These systematic evaluations included even unusual - some might say 'crazy' - ideas that were published and distributed in the web. It can be assumed that this 'open market' process was misinterpreted by 
many as indicating that all these ideas will ultimately also be part of DSM-5. As a consequence premature and ultimately unsubstantiated claims flourished.

\section{DSM-5 Did Tighten Up the Criteria - Sensibly and in the Interest of Patients, Not of Politicians!}

There is also the claim that DSM-5 is responsible for an artificial epidemic of mental disorders. Critics supporting this claim discard evidence that epidemiological rates of mental disorders are stable over the last 2-3 decades. They simply seem to dislike the sound of epidemiological numbers and the consistent finding that most of us will be experiencing at least one mental disorder in the course of our lives [Wittchen et al., 2011]. They obviously want us experts to fix this politically. How? By deleting certain diagnoses entirely and making the criteria - irrespective of their sensitivity, specificity, and utility - so stringent that numbers drop. Why should we do this in total absence of evidence? We have solidly known for decades that most patients with mental disorders are not recognized, diagnosed, and treated in time. Treatment almost always occurs much too late, typically years after onset, when the disease is chronic and multiple complications as a consequence of non-treatment make full remission unlikely. Thousands of papers provide conclusive evidence that the degree of suffering and the associated burden of disability of even a relatively small spectrum of mental disorders create a higher disability burden than any other disease group. Similarly, there is strong evidence for the effectiveness of early treatment, both from a trans-generational as well as a health economic perspective. Finally, some healthcare systems have actually tried this out, e.g., by providing care only for those with 'serious mental illness' (SMI), with little evidence for beneficial health economic or public health effects. So why tighten up criteria? Would internal medicine use stricter criteria for hypertension or diabetes simply to reduce the number of cases - at the expense of higher chronicity, disability, and mortality due to delayed treatment? These claims are clearly short-sighted and ridiculous, although they might perfectly fit in the competitive reasoning of some health economists, who have to allocate healthcare funds measuring impact only in terms of mortality rather than in long-term disability and quality of life.

\section{DSM-5 - Is There a Way Forward?}

Personally, I believe the primary value of DSM-5 lies in the tremendously complex and well-organized development process and I feel certain that we are on the right track towards future breakthroughs in our understanding of mental disorders. As this process towards DSM-5 has shown, the vast majority of existing diagnoses has sufficiently strong science and research support, with proven reliability, validity, and clinical utility for its users and their patients. This is a strong and encouraging outcome. That many, if not the majority of more 'revolutionary' and radical proposals for paradigm shifts failed in one, several, or all criteria of the rigid tests might be seen as disappointing. But this shows that we still have a long way to go in basic, preclinical, and clinical research as well as public health-oriented evaluations of innovations. Positively seen, the development process of DSM-5 has provided new perspectives and substantial guidance for future research. Will DSM-5 also reduce the harmful use of this instrument and facilitate the more frequent use of appropriate diagnostic procedures? I am afraid this will continue to be an uphill battle!

Hans-Ulrich Wittchen, Dresden

\section{References}

Phillips K, First MB, Pincus HA: Advancing DSM: Dilemmas in Psychiatric Diagnosis. Arlington, American Psychiatric Press, 2003.

Saxena S, Esparza P, Regier DA, Saraceno B, Sartorius N: Public Health Aspects of Diagnosis and Classification of Mental and Behavioural Disorders: Refining the Research Agenda for DSM-5 and ICD-11. Arlington, American Psychiatric Press, 2012.

Schumann G, Binder EB, Holte A, et al.: Stratified medicine for mental disorders. Eur Neuropsychopharmacol 2013; pii: S0924-977X(13)00276-9.

Wittchen H-U, Beesdo K, Gloster A: A new metastructure of mental disorders: helpful step into the future or harmful step back into the past? Psychol Med 2009;39:2083-2089.

Wittchen H-U, Höfler M, Gloster A, Craske M, Beesdo K: Options and dilemmas of dimensional measures for DSM-V; in Regier DA, Narrow WE, Kuhl EA, Kupfer D (eds): The Conceptual Evolution of DSM-5. Arlington, American Psychiatric Press, 2010.

Wittchen H-U, Jacobi J, Rehm J, et al.: The size and burden of mental disorders and other disorders of the brain in Europe. Eur Neuropsychopharmacol 2011;21: 655-679. 